\title{
ZUR CON.JUGATION.
}

\section{KUNPA UNI DAS T-PRAETERITUM.}

In Kölbings Englischen studien III, s. 160-163 in einer anzeige ron Kluges 'Beitrïgen zur gesch. der werm. conjugation' habe ich mich für ein germanisches 1 -prïteritum ausgesprochen.

Paul gelangt oben s. 136-152 am schlusse eines beitrags ' $/ \mathrm{ur}$ bildung des schwachen prïteritums und participiums' zu dem resultate, 1) dass 'alle diejenigen erklärungsversuche des schw. prït., welche für den dental des suffixes ein indog. $t$ voraussetzen, a limine abzulehnen sind', 2) dass es 'ungerechtfertigt ist für eine klasse der schw. präterita ein anderes suffix anzunehmen als fur die ibrigen', 3) dass 'alle scheinbaren schwierigkeiten, die sich der zurlickfuhrung des dentals auf indog. $d h$ in den wey stellen, sich auf befriedigende weise lïsen wie sonst bei keiner andern theorie'.

Dem satze 2 stimme ich vollkommen bei ${ }^{1}$ ), dem satze 3 widerspreche ich.

1) Hiermit soll gesigt sein, dass uns die gestalt des dentals in keiner weise nötigt, neben dem $t$-suffix ein $d /$-suffix anzusetzen, während die möglichkeit nicht ausgeschlossen werden soll, dass uns die

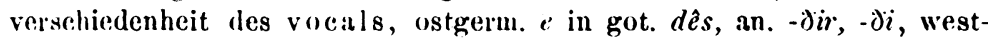

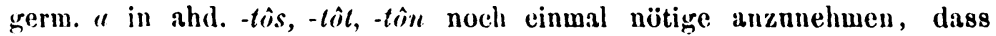
das ostgermanische den vocal eines urspriinglichen'-dhem = griech. $-\forall y, v^{\prime}$, das westgermanische $d \in n$ eines urspr. $-t \bar{a}^{\prime} m$, dessen $t$ im keltischen, dessen $a$ im lateinischen seine genauc entsprechung findet (s. Engl. stud. a. a. o. s. 1(i1), verallgemeinert habe. (Dass -dlièm künnte urspringlich den staken und schwichen verben, deren verbalstamm oder zweiter stamm auf einen vocal ausgeht (si siien etc.), das -tum den starken und schwachen verben anf consouantisch auslautenden verbalstamm (s. u.) 
Pauls bauptbeweismittel bilden (s. 150 f.) die präterita kunpa, unpa. Aus dem zusammenstoss von $n n$ mit einem $t$-laut ist entwedei germ. $n s$ oder $n$ ) entstanden. 'Woher nun aber', fragt Paul, 'die doppelte lautgestaltung? Diese frage', sagt er, 'kann mit voller sicherheit beantwortet werden. Wo suffix $-t i$ - zu grunde liegt, erscheint iiberall st, niemals $b$, vgl. kunst, brunst, ... ebenso dic 2. sg. kanst, -anst'. ' $n$ p entsteht aus $n n d h$ und nst a us nnt.'

Von der richtigkeit oder unrichtigkeit dieses sat\%es lasse ich für mich die entscheidung der frage nach einem grermanischen $d h$ - oder $t$-präteritum abhängen. Ist Pauls sat\% richtig, dann erkläre ich mit Paul, dass von einem germanischen tpräteritum nicht mehr die rede sein kann. Dann bin ich bereit, was ich bis jetzt noch nie getan habe, die gesanmiten germanischen präterita mit $h t, f t, s s$ als analogiebildungen nach dem participium anzuerkennen.

Aber der satz hat einen schwachen punkt. Paul selbst erkennt an, dass 'die lautphysiologische erklärung ibre schwierigkeiten hat'. Ich fordere jeden auf, der fur ein germanisches $d h$-präteritum ist, ernstlich zu versuchen, ob er für ein germanisches $n$ b aus nndh die erklärung finden kann. Ein übergang von nndh in $n f$ ist meiner ansicht nach nur dann möglich gewesen, wenn das urspr. dh zu germ. $\delta$, woraus nach dem nasal $d$, geworden ist auf demselben wege, den das $d / h$ inlautend im lateinischen zuriicklegte: $d h$ th $p d d$. Aber auch wenn wir dies annehmen wollen, ist nicht einzusehen, warum das dlh nach me auf der stufe $\phi$ beharte statt wie sonst in den tönenden laut iiberzugrehen. War dageucen, wie wir annehmen, die stufenfolge $d h \delta d$, war also der dentale geräuseh. laut auf dem zuriuckgelegten wege jederzeit tönend, und darun auch der vorhergehende nasal jeder\%eit tönend, dann ist nieht einzusehen, durch welches mitwirkende dritte element der dentale gerïuschlaut der tonlosen qualität teillaftig geworden sein kann.

Mit der doppelten lautgestaltung $n p$ und nst verhält es

zugekommen sein. Doch genïgt zur erklärung des ostgerm. ' die annahme eines starken prït. anf $-i m=$ griech. $-\eta v$, s. den folgenden aufsatz). Diese differen\% des vocals wird im folgenden nicht weiter berücksichtigt. [Vyl. über dieselbe jetzt Kügel, zs. t. gymn. 34, 40i.] 
sich nach meiner ansicht vielmehr so. $n n t$ wird $n p$ in urgermanischen oxytonen, $n n t$ wird $n s t$ in urgermanischer betonter stammsilbe. Die 2. sing. perf. kanst, anst trug den ton auf der stammsilbe, die wörter auf -li- wie kunst, brunst trugen, wenngleich ihr ablaut ursprunglich unbetonte silbe vorausset/t, dennoch in folge eines ausgleichs zum grossen teil den ton auf der stammsilbe. ${ }^{1}$ )

Ich unternehme es nun meinen satz nach der lautlichen seite zu begriunden.

Kögel hat oben s. 171-201, soweit unsere bekanntschaft mit dem urgermanischen accent es möglich machte, gezeigt, dass vorgermanisehes $t t$ (entstanden aus $t d d \dot{d}+t$ ), zwischen vocalen stehend, germ. $s s$ wird in oxytonen, germ. st bei betonter stammsilbe. $\quad-t t$ wird germ. (durch $* t h t, * b t) \quad s t .{ }^{2}$ ) Kögel irrt aber wenn er glaubt, $t t^{\prime}$ ' sei germ. $t t$ geblieben, ein

') Die wörter auf -ti- hatten ursprünglich nur in den obliquen casus reducierte stammsilbe, im nom. acc. betonte stammsilbe mit dem vocal o (germ. a). Später, als die beweglichkeit des accents verloren gieng, ward entweder der accent des nom. ace. durch alle casus fortgefiihrt, wie stets im griechischen, oder der der obliquen casus, und bei den meisten wörtern drang die reducierte gestalt der wurzelsilbe anch in den nom. ace. Eben so stand es urspriinglich bei den wörtern auf -lu-, nur bewahren die wörter anf -lu-, die den accent des nom. acc. verallgemeinern, etwas häufiger den diesen casus ursprïnglich gebiihrenden vocal der wurzelsilbe. - Got. ansts, ahd. as. anst, ae. est, an. ist wahren also die alte form des nom. acc. sing., während as. -unst, ahd. unst (masc., also ein wort auf -(lu-) den ablaut der obliquen casus anfweisen. S. hierzu im folgenden aufsat\% den abschnitt iiber die entstehung des 0 .

2) \%u streichen ist (s. 189) Kügels beispiel s, nhd. hast. Das wort ist uns aus dem französischen zugekommen auf dem unwege durch das mul. und innd. Sonderbarerweise wird die sache gewöhnlich umgekehrt dargestellt. Direct entlehnt aus dem altfranz. hisle sind nur das me. hast und das mnl. hacst ( $\mathrm{nnl}$. haast), aus dem mnl. ist das wort ins west- und ostfries. und ins onnd., aus dem mnd. ins nordische und ins hochleutsche gedrungen. Das franz. wort aber, mit dem adj. hastif, wo$\%$ ital. astivamente, ist mit dem regelmässigen roman. $a$ aus germ. ai das ins roman. gedrungene ahd. *heist $==$ ae. hếst f., wozn heisli vehemens (haistera handi lex Alam. = westfries. mit hacster hand), heistigo vehementer. Der ausfall des $f$ in diesem worte verglichen mit dem got. haifsls f. ist analog dem von Kügel s. 193 ff. dargelegten ansfall des $h$ im ahd. vor $s+$ cons. - |Zu no. 5, got. frasts, vyl. Kluge, KZ. $\mathrm{XXV}, 313.1$ 
'quantitïtslanger verschlusslaut' (s. 197), und sei cin tt gewesen zu der zeit wo die Römer Chatti, Nattium schrieben, ja dieses $t t$ habe sich auch noch in einzelnen nachziiglern bis in unsere älteren sprachlenkmäler gerettet. Wäre dies richtig, dann wäre dem $t$ in der tat nur eine kurze frist vergönnt, um auf dem ganzen germanischen gebiete ss zu werden. Aber schon zu der zeit, in weleher die Römer mit den Chatten verkebrten, kann der laut nicht $/$ gewesen sein. Denn wir besassen zu dieser zeit ein anderes $t$ in wörtern wie germ. slattuz schatz'): den quantitütslangen verschlusslaut dieser wörter hïtten die Römer ebenfalls durch $t$ widergegeben, von diesem aber muss der dental im namen der Hessen rerschieden gewesen sein. Vorgermanisches $t l^{\prime}$ ward germanisch (durch *thth) $\mathrm{zu}^{*} \mathrm{pb}$. Gedehnter spirant germ. p) konnte dem schicksal des einfachen $f$ vor betonter endung nach Verners regel, dem tönendwerden, nicht verfallen. Zu der zeit der Römer bestand dieses germ. $p p$, später ward es zwischen vocalcn gemeingermanisch ss. Wie hätten die Römer dieses germanische $p p$ in ihrer schrift widergeben sollen? Consequenterweise hätten sie thth schreiben mtissen, aber diese schreibung konute ihnen nicht anders als monströs erscheinen. Denn sie sprachen unter allen umstïnden $k, t$, auch wo sie $c h$, th schrieben. Wie sie nun häufig genug bei der widergabe der germanischen spiranten in der schrift das $\#$ sich ersparen und $c$ für grerm. $\chi, t$ für germ. $p$ schreiben statt $c h, t h$, so schrieben sie auch hier "t für germ. $p p .{ }^{2}$ ) Vgl. hiezu Mïllenhoffi, zs. f. d. alt. XXIII, 7.

1) (tot. sliatts, atta, smakka sind wol durch vermittlung des finnischen aus dem slavischen entlehnt (finn. $t$ aus $t$, ebenso dann finu. $k$ aus $\gamma$ in got. krêks, marikreitus).

$\left.{ }^{2}\right)$ tt vor der tunsilbe kunnte germanisch nicht "bleihen. llir vermutung, die ich selbst Kuhns zs. XXIV, j1 aufstellte, um ein rätselhaftes germ. $k$ aus urspr. $k$ vor der tonsilbe zu erkliiren, dass $k k \| \boldsymbol{l}\rangle$ im germanischen unverschoben bleibe, ist unhaltbar. Ich fand a. a. o. s. 459 ff. und 515 t. in zahlreichen wörtern an stelle eines erwarteten germ. $g$ aus urspr. palatalem $k$ vor der tonsilhe ein germ. $k$ und vermutete, dass eine dehnung des $k$ durch das ihm anhaftende $i$ der grumd der abnormen lautvertretuag sei. Die vermutung einer dehnung des $k$ lauts war richtig, nnr war dieselhe in der zeit falsch angesetzt: die deh. nung hat nicht vor der lautverschichung, sondern nach der wirkung visu Verners gesetz stattgefunden. Das richtige reigte, ohne jedoch auf meine sammlung rücksicht zu nehmen, Paul (Beitr. VIl, s. 133 ff. anm.). 
Unser germanisches präteritum erklärte ich Engl. stud. a. a. o. aus einem vorgermanischen imperfect (1. 2. 3. sing.) $-l \bar{u}^{\prime} m-t \bar{u}^{\prime} s-t \bar{a}^{\prime} \mid$ : germanisch setze ich dieses an als -ctān (mit nasaliertem rocal) - dās dā, da wir künftig germ. $\bar{a}$ und $\bar{o}$ werden zu scheiden haben '), doch kommt es uns hier nur auf die ursprüngliche gestalt des dentals, nicht auf den vocal der endung an.

Ahd. wessa ahd. as. wissa ae. wisse an. got. vissa 'wuste' ist bei der annahme eines 1-prïteritums regelrecht entstanden aus vorgerm. vittă'm vittä́t. Kögel erklärt (s. 186), wie es jeder muss, der ein t-präteritum ablehnt, vissa als analogiebildung nach dem alten participium viss und ahd. muosa 'muste' aus mōssa als analogiebildung nach ${ }^{2}$ lem part. ${ }^{*} m \bar{o} s s$. Aber jenes viss ist ein adjectiv, unser geviss, das zum verbum vait in gar keiner beziehung steht, das zwar in einer urzeit das particip zu vid 'sehen' war' (Kögel s. 172), aber niemals soweit unsere blicke reichen das particip des präteritopräsens 'gesehen haben, wissen' gewesen ist. ${ }^{2}$ ) Und das part. *mōss existiert gar nicht, es existierte nicht allein nicht in dieser gestalt sondern überhaupt nicht: in keinem älteren germanischen dialect begegnet ein part. pass. des präteritopräsens $m \bar{o} t$, in dem unsern, der das muosu besass, weder im ahd. noch im mhd., und wir durfen annehmen, dass die ältere sprache eines particips dieses prïtcritopräsens iiberhaupt nicht bedurft hat. Diejenigen, welche die präterita der präteritopräsentien als analogiebildungen nach den participien erklären, bitte ich uherbaupt zu

Paul find, dass ein nach der wirkung des Vernerschen gesetzes entstandener langer tönender verschlusslaut gremeingermanisch zum tonlosen verschlusslaut verschoben wirl. Die entwickelungsreihe filir mein beispicl germ. taikna war also nicht die a. a. 0. s.517 angesetzte, condern doicui-m, taizina, taiggna, taikikna, taikna.

1) S. den folgenden aufsatz.

\#) Unmïglich können wir es nicht nennen, dass viss eiumal als part. ¿u 'wissen' fungiert habe, denn es kommt ja zuweilen vor, dass ein adjectiv als particip des passivs zu einem ibm wurzelverwanten verb

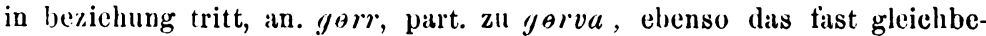
deuteude skr. pakvi-s gar, gekocht, fertig zubereitet, gereift, part. zu skr. pac. (Liese beiden wïrter sind gewiss reste eines urspriinglichen part. pass. aut $-v \dot{v}-)$. 
bedenken, wie selten nur die sprache sich genötigt sah ein particip zu diesen verben $\mathrm{zu}$ schaffen, wie viele dieser participien in den älteren dialekten gar nicht belegt sind. Man prüfe für unsere gegenwart das eigene sprachgefubl: wie häufig werden wir wol ein participium dieser verben bilder ausser zum ausdruck des perfects 'ich habe genust, gemust' etc.? (und wo die form gebildet wird, s. Paul, Beitr. IV, s. 325, da geschieht es jedes mal nach dem präteritum, nicht umgekehrt). Anders steht es mit den präteriten: diese müssen viel älter sein. Eines präteritums für vait $=$ foĩ $\delta \alpha$ hat es bedurft seit diese form präsensbedeutung hat. Diese präsensbedeutung ist aber uralt. Es muss also für unsere präteritopräsentien von der urzeit her zu jeder zeit eine präteritalform bestanden haben: diese muss in ibrer ältesten form und in ihrer grundbedeutung ein plusquamperfect gewesen sein. Unser schwaches präteritum baben wir in seiner form als ursprüngliches inperfect erkannt (s. Engl. stud. a. a. o.), unser präteritum der präteritopräsentien kanı also auch seiner form nach ein altes plusquamperfect sein. Sobald wir das imperfect des präsens durch ein mit einem dental gebildetes präteritum ablösten (s. Engl. stud. III, s. 161), werden wir auch das präteritum des perfects (dessen grundsprachliche gestalt sich aus den erhaltenen altindischen plusquamperfecten entnehmen lässt, s. Delbriick, Altind. verb. s. 121 f., Whitney, Ind. gramm. $\$ 817$ fí.), durch ein mit demselben dental gebildetes ersetzt haben. Unser munda ist also ein vorgerm. mem! $t \bar{a}^{\prime} m$ 'memineram', prät. zum

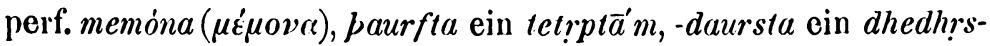
$t \bar{a}^{\prime} m$, und wir haben in diesen formen unser historisches plusquamperfect. Durch den verlust der perfectreduplication fiel das plusquamperfect der starken verben mit dem prïteritum zusammen.

Von unserm kann lautete, wenn wir das nn wie es vorliegt der zeit vor der lautverschiebung zuschreiben dürfen, das prät. vorgermanisch *gegnnlă $m$, das feminine verbalsubstantiv, dessen wir uns gewiss wie die östlichen nachbarn als infinitivs bedienen konnten, vorgerm. * gn'nti-s. Das consonantische $n$ ward vor dem tonlosen $\ell$ tonlos, $N$ (s. Hoffory, Kuhns zs. XXIII, s. 546 ff., Zs. f. d.a. XXII, s. 374 ff.), und es widerfuhr diesem tonlosen $N$ nach dem selbstlautenden $n$ was tonlos ge- 
wordenem nasal vor tonlosem verschlusslaut im nordischen stets begegnet, $N t$ ward $t$. $N t$ und $t t$ unterscheiden sich nur darin, dass bis zur sprengung des verschlusses das gaumensegeì hier emporgezogen, ciort gesenkt ist. Wir gewinnen so prät. gegnttă $m$, subst. g!'tti-s. Langer verschlusslaut, wie er auch nach consonanten häufig genug begegnet, kann nach sonanten die nicht vocale sind genau so gut wie nach vocalen seine stelle haben, man denke z. b. an wörter wie skr. vrtti-s die 'werdung'. Nach Kögels hinsichtlich des germanischen lautzustandes berichtigter regel uber vorgerm. It nun ward jenes präteritum germ. kunppä́n, dieses subst. kúnsti-z. Die länge des $p p$ unterlag der verkürzung nach vorhergehendem consonanten $\mathrm{zu}$ einer zeit, in der Verners gesetz sich bereits vollyogen hatte, diese verkiirzung zu $b$ entzog das $p p$ dem übergang in ss.

Kögel selbst lehrt ausdriicklich: 'ss war urgermanisch immer intervocalisch'. S. 199 meint er, da nicht intervocalisches ss 'nicht existiert und auch eine andere vertretung dafur nicht nachweisbar ist, so muss das geset\% gelten: indogerm. $d d h t+t$ erscheinen in nichtintervocalischer stellung im germanischen stets als st und sind vom accente unabhängig'. Aber Kögels beispiele für st aus $t t$ nach consonanten haben alle ursprünglich betonte stammsilbe, got. gilstr, 2. sing. perf. varst ${ }^{1}$ ), auch hurst strauchwerk ist als aus $k r^{\prime} t t i-s$ entstanden anzusetzen. Seine regel ist also ferner zu berichtigen: -' $t$ wird stets $s t$, $t t^{\prime}$ wird intervocalisch germ. $s s$, nach liquiden und nasalen gernı. $p$.

Bestand vor der lautverschiebung ein $u v$ statt des spätern germ. $n n$, dann ward in *geg!vtä $m$, ${ }^{*}$ g! $!^{\prime} v t i-s$ das $v$ vor $t$ tonlos also zum laut des neuengl. wh (keineswegs war v mit dem tönenden labialen spiranten identisch und ward $f$, wie Verner ZDA. XXI, s. 431 annimmt). Auch das so sich ergebende $V t$ ward $t t$ : das tonlose $V^{\prime}$ gestattete es dem $t$ gewis noch leichter als das $N$ seine articulation $z$ anticipieren, um selbst dann im $t t$ sich zu verlieren. Wir werden dieser auffassung den

') Ald. gersta ist abor gewiss gheirzdhä- (zu ghrs 'horrëre') wie an. prọstr drossel trozdlu-, vgl. lit. stra'zda-s (zdh zu st, wie zgh zu sk in den aussergotischen formen von $a z g i$ ). 
vorzug geben wegen des dieser selben erklärung (Iveniger leicht eincr der ersten analogen) sich fügenden mhd. wulst aus $v l^{\prime}$ 'ti-s aus $v l^{\prime} v t i-s$ (Verner a. a. o. s. 434) und des wahrscheinlich ebenso entstandenen ahd. swulst zu swellan. $\left.{ }^{1}\right)$

Pauls frage (s. 149 anm.) ob von -ginnan das starke oder das schwache prät. ursprünglich sei, geht von der unrichtigen voraussetzung aus, dass urspriinglich jedem verbum nur entweder das starke oder das schwache prät. hahe zukommen können. Unser sogenanntes schwaches präteritum ist ein imperfect, unser sogenanntes starkes das alte perfect, folglich kam ursprünglich jedem germanischen verb, (lem primüren wie dem abgeleiteten, eine jede der beiden bildungen zu. Denn dass auch fürs germanische ein älterer zustand vorauszusetzen ist, in welchem nach form und function unterschieden ein inperfect und ein perfect neben einander bestanden, kann von keinem ernstlich bezweifelt werden. Von -ginnan sind uns beide formen erhalten, in got. dugumn ac. -zon as. bi-gan etc. das perfect, in ahd. bigonda, bigunda, mhd. gunde, mnd. begonde, begunde, westfiries. bigonde, me. bè-gùde (schott. hêgouth) das imperfect.

Wie aber erklären sich nun die auf dem gebiete des deutschen auftretenden präteritalformen mit nst, ahd. Otfi. konsta, gionsta, md. Jiilich kunste (Weinh., mhd. gr. 392), as. Hel. consla, gi-, af- onsla, mond. nicht zu finden; ahd. Isid. bigunsta, md. türing. begonste, begunste (Weinh. 381), as. (boichte)

1) (Nachgetragen.) kann ist das perfect nicht zu einem prijsens auf $-n-u$, sondern $z u$ cinem auf $-n-A$ (d. h. der dritte radicale consonant ist nicht $u$ wie in kr$u$ hören, sondern $A, g n d$, s. Saussure, Syst. prim. s. 2ff ff.): das prïisens liegt vor in sanskr. 8. Saussure s. 25(i) aus gnni' $m i$, und wahrscheinlich im gotischen selbst in kunna $\gamma \iota \gamma v(\dot{\sigma} \sigma \omega$, das eben dieses starke verb wire, in die schw. conj. eingetreten. Das femin. kunst ist also "gn'nA-ti-s, eine bildung wie $\delta_{i} v \alpha-\sigma l-\varsigma, \delta^{\prime} v \alpha-\mu t-\xi$, das particip got. kumps ist "gnnA-to-s, eine bildung wie $\delta \imath v a-\tau \dot{c}-\varsigma$. Das $A$ (dessen lautwert wol der spiritus lenis, s. den folgenden aufsatz) schwindet im germanischen spurlos, wie in got. duuhtar

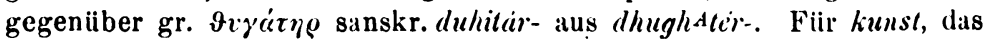
part. kunps und das prät. kunfa, in welchem wie in kann das $n$ des präsens ins perfect drang, ist also die erste erklärung des ntt aus mint herzustellen, für die $n n$ aus $n v$ (wie in rinnan) und die $l l$ aus $l v$ aber hat die zweite des $n t l$, llt aus $n v t$, lvt zu gelten. 
higonslu, mund. (Münst. chron.) hegues/. (Das altfrics. Riistr. higonste muss, weil es den fries. lautgesetzen widerspricht, notwendig aus dem nd. eingedrungen sein.). Nienıand wird umıiin können in diesen formen analogiebildungen $z u$ sehen. $\mathrm{Zu}$ ihrer erklärung bieten sich mehrere möglichkeiten.

Das präteritum kann sich nach dem alten infinitiv, einen casus der verbalsubstantive kunst, ahd. as. -unst gerichtet haben. Dass dieser infinitiv einmal in der gestalt kúnslai, mundái, skuldái, máhlui etc. im germanischen bestanden habe, lassen die altnordischen infinitive mundu myndu, skyldu, máttu schliessen (zum letzten vgl. die anm., das $y$ in myndu, skyldu stammt aus dem priit. und in diesem aus dem optativ): die feminina auf $-i$ sind im nordischen im sing. in die $\bar{u}$-declination iibergetreten, wie $z$. b. an. $g^{\prime}$ st aus ansti-, das - $u$ ist die endung des dativs der $\bar{a}$-declination, welche die stelle des casus der $i$-declination eingenommen hat. Wir werden die entstehung der deutschen präterita mit nst schwerlich bis in die lebenszeit dieser infinitive hinaufrucken dürfen, aber auch noch in jüngerer zeit konnte sich das prät. zu kann, ann einfach nach den verbalsubstantiv umbilden, konsta zu kunst wie scolta zu scult, dorfta zu durft, gitorsta zu turst. $\left.{ }^{1}\right)$ Otfrid hat sonst konda, onta: das einzige mal wo er fiur jenes die $s$-form braucht $(\mathrm{III}, 16,7)$, ist es in folgendem satze: 'ioh sinera kunsti, unio er thio buah konsti', ebenso steht gionsti, zu dem er nur noch einmal den indicativ hat, im reim auf ensti $(\mathrm{V}, 25,101)$.

Diese erklärung der st-formen des prïteritums aus dem st des verbalsubstantivs halte ich für die zutreffende. 'Zugleich nit diesem substantiv kann dic grestalt der consonantengruppe

1) Ebenso verdankt das prät. got. ahd. as. mahta afr. machte ae. meahle an. mailla, neben dem ïlteren ahd. as. mohta, sein a, das dann zum teil auch in den plural got. mayum etc. drang, gewiss nur dem zufïlligen uustande, dass das subst. die gestalt germ. mahti-z (vorgerm. moliti-s, slav. mośtr) batte (das vorgerı. mo- anstatt des $m$ der obliquen kasus ist nach dem folgenden aufsatz zu heurteilen). Das dïnische, das allch in skulle (ll phonet. schreibung fiir histor. ld) und andern formen denselben alten infin. festhäl, hat in seinem (mit der prïposition verbundenen) at mâtte den dem slavischen mösı entsprechenden alten intinitiv mahtai. (In der lebenden sprache ist at maite gelaiufig und unentbehrlich, wenn auch in schriften relativ selten.) 
in der 2. sing. zur entstehung des st beigetragen haben: prät. mohta, -lorsta, dorfta neben 2. sing. maht, tarst, darft, also prät. konsıa, -onsta zu lanst, -anst, und ebenso fränk. unista $\mathrm{zu}$ uucisl, as. etc. môsta zu môst.

Möglich wäre auch die folgende entstehung des st. Wie altsächs. môsta für älteres * môssa das $t$ eingefügt hat nach der analogie von gi-dorsta, thorfta, mohta, êhta und den zahlreichen andern prïteriten mit ht (Kögel s. 186 anm.), so kann gleichzeitig ein älteres *konpa durch einfügung desselben I konsta geworden sein. Denn ein *kompta konnte sich keinen augenblick balten, sondern muste sofort noch bevor das wort zum ersten mal in den mund genommen ward, sein $p t$ in $s /$ verkehren, nicht durch lautgesetzlichen wandel, sondern durch nachbildung zahlreicher anderer offenbar die stelle von $p+t$ vertretender $s t$, die es ins sprachbewustsein übergehn liessen, dass $p+t$ st ergibt, dieselbe nachbildung geläufiger vorbilder, welche verschiedene $k t$ jiungerer entstehung im deutschen, altfriesischen 1) und altenglischen in ht gewandelt hat.

Paul (s. 151) wendet sich, um die präterita mit nst zu erklären, an die participien: bei diesen lässt er das nst lautlich entstehn. Aber es geht unmöglich, wie es Paul tut, das gemeingermanische part. got. kunps, kund, notus für eine analogiebildung nach lem präteritum zu erklïren: kunps ist, weil adjectiv geworden, notwendig alt, es ist das regelrecht gebildete particip vorgerm. ${ }^{*}$ g! tt $\dot{0}-s$, iber welches s. 464 anm. Man könnte, wenn man Pauls weg der erklärung des nist aus dem particip einschlagen will, annehmen, man hahe einmal vor der lautverschiebung, um die participien von den adjectiven zu scheiden, bei jenen den accent verriickt (barytonierung des -10fiudet sich ja im germanischen auch sonst), also vittó-s adj. 'gewiss', vitto-s part. 'gewust' (mhd. gewest, gewist), ebenso gnttó-s adj. 'kund', gn! tlo-s part. 'gekonnt' = gewust. $\left.{ }^{2}\right)$ Aber die sache ist misslich, weil die participien *kunst, *unst gar

1) Das nordfriesische jedoch hat das ht genau innerhalb seiner alten grenzen gelassen, ebenso wie das nordische sein entsprechendes $t$.

$\left.{ }^{2}\right)$ Doch wäre dann eher zuriickziehung des accents bei den adjectiven zul erwarten gewesen. Vgl. skr. rikita-s leer, zuista-s angenehm, part. von ric $\lambda \varepsilon i \pi \omega$, $z u s ~ \gamma \& v \omega \omega$. Die erklïrung dieser "zuriickziehung des accents' wird der folgende aufsatz bringen. 
nicht uberliefert sind, das mol. tiiring. begonst, begunst dagegen (das eine analogiebildung nach jenem *konst, *kunst sein miiste) und genau ebenso das bair. gewest alem. mil. gewist (s. Weinh.) whe allen zweifel junge biidungen nach dem priteritum sind, nicht solche nach denen sich das prät. hätte bilden können.1)

Pauls satz ist also beseitigt. kunps ist eine alte bildung auf $-t \dot{o}$-, für welche umbildung nach einem präteritum mit $d h$ nicht zugegeben werden kann, und kumpa kann kein dh-prïteritum sein. kunpa ist sicher und notwendig eine nur bei annahme eines ursprünglichen $t$ für den dental erklärbare form genau so wie die lange reibe der präterita mit $s s, h t, f t$. Dem anhänger des $d h$-präteritums und des nach dem particip gebildeten analogiepräteritums bleibt also auch für kunpa kein anderer weg als der, es als analogiebildung nach kunps zu erklären, wie er vissa nach viss erklärt. Aber kunpa ist weit entfernt davon, eine stütze, als welche es Paul s. $150 \mathrm{f}$. verwendet, für die ansetzung eines $d h$-prïteritums abzugeben.

Das durch verkümmerung seiner lebenshedingungen schon ziemlich mitgenommene analogiepräteritum soll sich der genugtuung bei kun $b^{\prime} \iota$ mit dieser aussicht auf lebensfristung davon gekommen zu sein nicht lange erfreuen. Denn kumpa hat noch einen genossen: bei diesem ist die analogicbildung nach dem participium ausgeschlossen, denn das participium, das hierzu nötig wäre, existiert gar nicht. Es ist dies das prït. an. olla zu valda walten, part. valdit.

olla (opt. ylla), in gotischer form *valpa, ist vorgermanisch

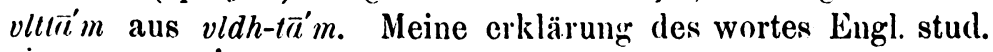

1) Eine fernere denkbare erkliirung des $s t$ ist mir erst nachtriiglich ringefallen. Dio priiterita unpa und gunpa waren von alters her mit priipositionen verbunden, sie könnten in diesem falle den accent urgermanisch zurïckgezogen haben, daher un/a aber ga-unsta, gun/a aber bi-gunsta, mössa aber got. ga-mòsta. Altsïichs. etc. consta miiste nach der analogie von gi-onsta und li-gonsta gebildet sein. Aber mit der urgerm. betonung der mit präpositionen verbundenen verbalformen ist es eine ziemlich sehwierige sache, die noch sehr der untersuchung bedarf. Altsächs. af-onsta wïre germanisch vor der verschiebung gewesen apo n'tlam, verschoben alj-instän, mit der germanischen accentverschiebung wïre dann bei den in historischer zeit betonten präpositionen der accent wider zurückgezogen, as. if-onsta, während er bei ga- etc. blieb, as. gi-onsta. 
III, s. 161 war nicht die richtige, der wurzelauslaut ist nach dem slavisch-litauischen $\left.d h .{ }^{1}\right)$ An. olla heweist die richtigkeit meiner bchauptung uber die vertretung von vorgerm. $t$ im germanischen, und bestätigt die gregebene erklärung von kunpa. Wie will man olla als $d h$-präteritum erklären? Hat Paul auch lafuir einen satz 'dhdh wird $p$ '?

Neben diesen vom nordischen bewabrten präteritum bestand natiirlich im germanischen das perfect, das die ubrigen germanischen dialecte festgehalten haben. Das präsens des verbs valdan ist nach Kluge, Germ. conj. s. 155 ff. anzusetzen als ein urspriungliches auf $-m i$, vorgerm. vóldhmi, plur. vldhmé. . (vgl. den ïbergang des ältern $-m i$ in juingeres $-i$ im litauischen). Das germanische verb hat wie das slavische cinen präsensstamm, und einen nichtpräsens- oder zweiten stamm, ausserdem als dritten den perfectstamm. Vom zweiten stamme wird wie in slavischen gebildet das verbalsubstantiv auf $-t i$, also der alte infinitiv, und las schwache präteritum.

Bei zahlreichen starken verben ist der präsensstamn un das element -jo-reicher als der zweite stamm, von lem das $t$-prät. gebildet ist. Zunächst bei den starken verben consonantisch auslautender wurzel sôkjan, rôkjan, bugjan, vaurkjan, punkjun. Die beiden letzten haben in juingerer zeit in einzelnen dialecten an die stelle des $u$ der abgelauteten wurzelsilbe im präsens das $e(i)$ der nicht abgelauteten treten lassen, as. nirkean, fränk. wirken (wic gr. 'óç(1)) neben obd.wurchen, ebenso ae. pincean an. piklija neben pykkja: das so entstandene verhältnis wirkjan worhta, pinkjan * punhta belehrt uns, dass zu der zeit in welcher das präsens umgebildet ward, zahlreiche starke verben auf -o- mit dem vocal $e(i)$ in präsens von der art wie -yinnan ein prät. wie -gunpu gehabt haben misssen.") Dass im altsächs. das prät. zu wirkean den vokal $a$ annahm,

1) Es gibt zwar, wie ich glaube, im slavischen mehr lehnwörter aus dem germanischen als angenoumen wird. Aber meiue annahme, der wurzelatuslaut unseres verba sei urspr. $\iota$, griindete sich nur anf an. olla. Das altir. flaith f. herschaft stammt von einer kiirzeren des $d / h$ entbehrenden wurzel.

2) Ein andere erklärung des $e$, woraus $i$, in pincean, wirkean $=$ ó $\dot{c} \zeta(\omega)$, die der hier gegebenen vorzuziehen ist, sowie des nicht reducierten vocals in sokjan, rojkjan, flokjan $=\pi \lambda . j \sigma \sigma \omega($, s. im folgenden aufsatz. 
warahta, lehrt uns, dass als dies geschah neben dem präteritum ein perfect :wark, wie bi-gann, bestand.

Ferner aber besteht dasselbe verhältnis zwischen präsensstanım und \%weitem stamm bei nicht wenigen starken verben auf auslautenden langen vocal. Wurzel $n \bar{e}$ nähen, $s \bar{e}$ säen, $r \bar{e}$ wehen, gne kennen (gr. lat. gni slar. $z n \bar{a}$ lıaben den vocal des perfects und eines urspr. prïsens gignī-mi verallgemeinert) u. s. w., präsensstamm nējo- genau wie in lat. neo, präsens rorgerm. $s \vec{e}^{\prime} j e-t i, v \vec{e} j e-t i$ genau wie im slavischen, rom zweiten stamme das prïteritum vorgerm. ${ }^{*} s \bar{p}-t \bar{a} m$, an. sáða, knáda, ahd. sâta, wâta, nâta ${ }^{1}$ ), gebildet ganz wie lat. nêe-bam, und das rerbalsubstantiv, unser sât, nât. Das neben dem präteritum bestehende perfect bildeten diese rocalisch auslautenden verben alle mittels eines $v$ (iiber ein $v$-perfect s. Engl. stud. III, s. 162 unten), wie lat. nīvi, sèvi, nēvi: 3. sing. vorgerm. sesī́ve, got. saisô ae. séow as. (otar-)sêu Cott., gegnō've, ae. cnéon. Das $v$ des perfects ist im ae. sâwan, cnâwan etc. und \%. t. im hd. ins präsens gedrungen. Das st der 2. sing. got. saisôst ist aus $-t t$ aus $v t$ entstanden.

Das verb $d h \bar{e}$ 'tun' hatte im germanischen ein doppeltes präsens, urspr. (dhi)dho'ti, ae. $d \bar{e} p$ ahd. tuot, und urspr. $d h \vec{e}^{\prime} j e t i$ = slav. $d \bar{e}^{\prime}$ jet $\grave{\imath}$ ponit: diese präsensform ist erhalten in der 2. 3. sing. mnd. nnd. deist, deit, rgl. seit, weit etc. aus dem oben angesetzten séjeti, geit (Otfrid geist, geit) aus vorgerm. ghè'jeti (neben gàt aus ghe'ti). Zum ersten präsens gehört das imperfect dhidhóm, ac. dide as. deda ahd. teta, plur. ac. didon as. dedun. Vom zweiten stamme $d h \bar{e}$ - ist gebildet der schwache aorist *dhê-t $t \bar{a}^{\prime} m$, vorliegend in altfries. dêde und dem plur. ae. dî̀dun as. dadın ahd. tâtun. * dhề $\vec{a}^{\prime} m$ verhïlt sich

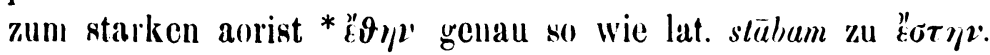
Der sing. ahd. tela ist also starkes, der plur. tâtun schw. prït. Diesc erklärung der pluralform unseres prät. ist, glaube ich,

1) Diese $i$-losen furmeu (mhd. sàte, wâte) sind älter als die nach der amalogie der ersten schwachen conjugation mit heriibernahme des $j$ des prïisensstammes gebildeten sâta $=$ as. sinida (mhd. nhd. sîte $=$ nd. scide). Als kur/silbige verben (denn wur\%elhafic auslautender langer vocal steht wie scinem ursprungr so seinem zeituass nach jederzeit kurzew vocal + cons. gleich) hiitten sie in der ersten schwachen conj. ihr $i$ des präi. nicht aufigeben können. 
der ziemlich complicierten vorzuziehen, die ich Engl. stud. III, 155, 163 aufstellte. Der sing. afr. dêde (mit sicher langem $\hat{e}$ ) mag iibrigens eine jüngere analogiebildung nach dem plur. sein, wie uuser nhd. tât. Vom zweiten stamme ferner das verbalsubstantiv $d h \bar{e}-t i-s$, got. dêds ete. = slav. dêt $t \bar{t}$, inf. slav. détī. Wäire unser substantiv eine ursprüng̣liche, indogerm. bildung, daun miiste die form kurzen vocal haben wie griech. $\vartheta \dot{\sigma} \sigma \varsigma$, die form ist also erst in nachgrundsprachlicher aber vorgermanischer zeit aus dem nordeuropäischen zweiten stamme zum zwecke der verwendung als infinitiv geschaffen. Die rerben dhe und ghe haben das particip auf -no-bewahrt, altfries. dèn und gên ete. = slav. dênŭ: wir haben also bei dhe wider cinmal ein schwaches präteritum ohne daneben bestehendes particip auf $-t \dot{o}$ -

Um ein -jo- reicher als der zweite stamm ist der präsensstamm bei den abgeleiteten verben auf - tjan $=$ griech. $-\zeta(\omega$ (s. Kögrel s. 183), wie got. kaupatjan. Der präsensstanm greht aus auf urspr. - $d$-jo- germ. -tja-, der zweite stamm auf urspr. $-d-$ Das prät. lautete also vorgerm. $-t-t \bar{a} m$, was wenn das suffix betont war, got. -ssa geben muste, also *kaupass ist wol anzunehmen, dass das gotische in kaupasta genau so wie in môsı nach der analogie der zahlreichen präterita auf $s t, f l, h t$ das ss durch st ersetyte. Sonst müste dem got. -stl ein - Häm mit betonung des vorhergehenden vocals zu grrunde liegen. Ein participium auf -ss oder -st, von welchen die anhänger des analogiepräteritums das prït. kaupasia herleiten kïnnten, findet sich nicht. Darum wird (kaup)-asta in der regel ron den anhüngern jener theorie ignoriert und fur eine grotische neuerung erklïrt.

Zwei germanische verben mit dem präsensstanım auf'-c'-johabeu geneingermanisch consonantisch auslautenden zweiten

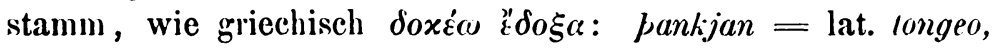
prät. pâhta (aus lonkt $\bar{a}^{\prime} m$ ), und branyjan. Das prät. brâhta gehört nicht ursprünglich zum starken verb bringan, denn von diesen könnte der schwache aorist nur *brûhıa lauten, es müste sonst, was nicht wahrscheinlich ist, schon fiuhe, gemeingermanisch, das " aus dem perfect brang eingedrungen sein, wie es in altsächs. warahta aus dem perfect eingedrungen ist. In die analogie von pankjun und brangjan sind in jüngerer 
zeit in westgermanischen, nicht in gemeingermanischen, die causative auf -akjan (oder gemeinwestgerm. -akkjun) cingetreten, wakjan wahtu (ahd. as. muhhta ae. weahte), pakjun pahta (ahd. -duhtu N.. doch s. Paul s. 140, ae. peahte), rakjan rahtu (ahd. -rahta ae. reahle). Die unmittelbare anfïgung ler suffixe des prïterituns und des particips an den auslaut der wur\%cl sagte keinen andern verben so sehr zu als denen mit einem $k$-laut als wurzelauslaut. Die zugehörigen participien vergleichen sich dem lat. doctus zu doceo. Urspriinglich sind solche formen nicht, denn dem causativ kam urspriinglich im zweiten stamme und im particip überall ein $-i-$ zu. ${ }^{1}$ ) Das gotische und nordische wissen ron diesen formen nicits, die nordischen präterita lauten nicht * vátta, * pálta.

Ferner sind im westgermanischen in dieselbe analogie die causative auf -aljan eingetreten, prät. salda, lalda, dwalda, kwalda (die formen s. bei Begemann s. $120 \mathrm{ff}$., Paul s. 1411). Dass diese formen auch nordisch gewesen seien (vor der nordischen syncope, welche alle kurzsilbigen ihnen gleich machte), ist nicht nachzuweisen.

Dic genannten westgermanischen formen auf -ahtu, -alda, part. -aht, -ald sind sehr junge analogiebildungen, denn wir sehen sie noch vor unsern augen in bestïndigem schwanken wit den lautgesetzlich berechtigten formen auf -ekida, -elida, lart. -ekid, -elid, und sie haben auch in der folge keineswegs auf dem ganzen gebiete des westgerm. iiber jene ïlteren und ïber die später aus der analogie der langsilbigen ihnen erwatchsenen nebenbuhler den sieg davongetragen, während die alren tiberkommenen seit gemeingermanischer orler noch friiherer zeit keine nebenbuhler kennenten prïterita pahta, sôhta festslehil. Ein anzcichen der jugend fiir die -ahla, -alda ist, dass ilmen keine von dem gleichen consonautisch auslautendeu

1) Die verba pankjan und brangjan sind nicht als ursprüngliche calusative anzusetzen, was auch ihre bedeutung nicht fordert. Sind franlijan und brangjan urspriingliche casusative gewesen, denen von alters her das particip auf urspr. -i-li-zukam, dann ist anzunehmen, d:ass süc im urgermannischen durch die nahe beriihrung mit den ihnen verwamten starken verben purkjan und bringan zu ihrem vocallos gebildeten prïteritum und particip, und pankjan zu seinem verbalsubstantiv ahdid. anuelialt, gelingt sind. 
zweiten stamme gebildeten verbalsubstantive auf -ti-, also keine alten infinitive zur seite stehen, wie den alten formen dieser bildung.

Die präterita ae. wehte, pehte, rehte, von denen ich nicht mit Paul glauben kann, dass ihr $e=e a$ ist, sind compromissbildungen zwischen den rerschiedenen mit einander kïmpfeuden wekede, weuhte, wecte. $\left.{ }^{1}\right)$

Alle andern durch mangel des umlauts aus ihrer klasse heraustretenden präterita zu causativen ausser solchen auf den wurzclauslaut $k$ oder $l$ sind noch jüngere bildungen: sie tauchen nur in einzelnen westgermanischen dialecten anf, keines derselben erstreckt sich iiber das ganze westgermanische sebiet. Von diesen unten.

Nur im $h t$ des prät. und part. für $k t$ folgt der analogic von ahd. suochan suohta, altfries. sêka sochte, ae. sêcean sohte das causativ 'reichen' ahd. reichan reihla, altfries. rēka rachle, ae. rî́cean rahte (ebenso das causativ ae. thcean tohle). Im altengl. prït. sehen wir den umlaut, und Paul ist im irrtum, wenn er annimmt, dass im altfries. prït. rachte der umlaut fehlt. Denn altfries. $a$ (aus $\ddot{a}$ ) ist regelrechte verkürzung des altfries. offenen $\vec{e}$ (= got. ai und umlaut von altfries. $\hat{a})$, vor consonantengruppen wie in altfries. $\bar{e} n$, acc. anne unum, nanne nullum, famne, ham-merke dorfmark, hladder leiter, askia, flask, mas/ meist, hast vehemens (ahd. heisti), lasta leisten, gastlik geistlich (neben Rustr. i'sllik: die verkürzung unterbleibt vor st im wescrfriesischen und in dem einen dialekt des nordfries. genau wie im neuengl. most, ghost, east), während vom altfries. geschlossenen $\hat{e}(=$ rot. $\hat{e}$ und umlaut von $\hat{n}, \hat{u})$ die kurzung $e$ ist.

Von den verben der \%weiten sehwachen conjugation (ahd. $-\hat{e} n)$, die der griechischen auf -óo entspricht, mit dem prïsensstamm auf friberes -o-jo-, hahen cinige, und zwar die geclüufigsten kurzsilbigen, in westicermanischen offenbar alte formen des prïteritums ohne mittleren vocal gewabrt, wclche zn den formen des nordischen stimmen, das die alte flexion unserer klasse am getreuesten festgehalten hat. Altsïchs. andfrk.

1) Die altnord. pritterita scldla und sella, part. seldr nnd settr, halte ich für reste einer germanischen i-conjugration (= lat. impf. älter -îbam, part. -îlus), selda aus germ. * saliula", séldr aus * salida-z. 
habda, hadda, ahd. Is. Frg. hapta, mhd. md. hatte mit dem opt. helle, wozu unsere nhd. form, wegen des umlauts im opt. sicher ursprünglich zweisilbig, ae. hxfde $=$ an. hafda; as. anfrk. sayda, ae. sazde $=$ an. sagða; as. libda, ae. lifde $=$ an. lif $\partial a$. $\mathrm{Zu}$ diesen muss auch gehören als ein prät. derselben klasse as. hogda, plur. hugdun, ahd. hogta 0, -hocta, ae. hozde. Das präsens lautet zwar got. hugjan an. hyggja ae. hyczan, aber ein austausch zwischen den schwachen conjugationen findet sich ja mehrfach, wir haben ebenso an. fylgja ae. fylzan neben ae. folzian ahd. folgên (dazu lat. taceo neben got. pahan u. a, s. wegen dieses wechsels der conjugation den folgenden aufsatz). Das prät. hogda sieht dem sagda etc. völlig ähnlich, als prït. der ersten schwachen conj. aber kann ich es nicht erklären. Paul stellt hugjan auf eine linie mit dem starken verb bugjan, und erteilt diesen verben ein particip boht, *hoht, und ein prät. *bogda, hogda: der dann erfolgte ausgleichungsprocess hätte diese schöne congruenz zerstört. Ich glaube nicht, dass sich unter den ursprunglich beiden verben in gleicher weise $\%$ gebote stehenden formen ein solcher ausyleichungsprocess nach verschiedenen richtungen hin hätte vollziehen können. ${ }^{1}$ ) Paul hält das prät. hogda mit sagda und genossen für einen sicheren zeugen eines $\boldsymbol{d} h$-präteritums. Die formen sehn freilich selur nach $d h$-präteriten aus. Ist denn aber Paul bei hogda nicht das verbalsubstantiv got. gahugds ae. zehyzd f. in den sinn gekommen? Dies substantiv mliste sein $g d$ für erwartetes ht demselben ausgleichungsprocess verdanken. Holtzmann sagt s. 50: 'auffallend ist gahugds, das vielleicht bleibt, weil

1) Es miiste (vgl. Yaul, Beitr. IV, s. 324 fi.) einer von bugjan nur das part. lowh, dagegen von hugjan nur das prät. hogda gehört, und dann, da für ihn die verben nicht einauder congruent waren, die noch nicht gehörten furmen nach den schon gehörten gebildet haben nach dem von zahlreichen andern verben her bekannten verhältuis zwischen präteritum und particip. Dies könnte vereinzelt leicht vorgekommen sein, aber das geniigt noch nicht, deun es hätte keine nachahmung bewirkt: es miiste unzählige male vergekommen sein, und von bugjan inïste das präteritum, von hugjan das particip eine lange zeit hindurch selten oder nie gebraucht sein, bis eine jüngere zeit, die der einen der beiden oder zugleich beider formen wider bedurfte, dieselben nun ohne riicksicht auf das andere lïngst nicht mehr analoge verb nach den der zeit geläufigen analogien bildete. 
gahauhts zu weit abstünde.' Kluge, Conj. s. 121, bemerkt: 'sicher scheint zu sein, dass ein kugh-tis zu grunde liegrt und dass $g h+t$ sonst stets durch $h t$ reflectiert wird.' Dass gahugds ein urspr. I enthält, hat noch niemand geleugnet: könnte das $d$ ein $d h$ sein, dann wäre das wort schon längst nicht mehr rätselhaft gewesen. Das verbalsubstantiv gahugds beweist auch für das gotische ein älteres präteritum *hugda. Lin anderer ursprünglicher infinitiv unserer conjugation steckt in dem subst. ahd. dult as. githuld ae. zepyld zum verb got. Julan an. pola ahd. dolên, wir erschliessen aus demselben ein älteres prät. ${ }^{*}$ pulda $=$ an. polda. ${ }^{1}$ )

Untersuchen wir nun, welchen grund das gd in gahuyds: hat. Wie musten die urspriinglichen präterita und verbalsubstantive unserer zweiten schwachen conjugation lauten? Der prïsensstamm ist bei vielen starken verben, in der schwachen $\bar{a}$-conjugation, in der slavischen schwachen $\bar{e}$-conjugation, um ein $-j o$ - reicher als der zweite stamm ${ }^{2}$ ): der frühere ausuang des präsens war in unserer eonj. 1. sing. $-0-j \bar{o}, 3$. $-o-j e-t i$, wie in der (dritten schwachen) $\bar{a}$-conj. 1. $-\bar{a}-j \bar{o}, 3 .-\bar{a}-j e-t i$, wir dürfen also vermuten, dass der zweite stamm unserer zweiten schwachen coujugation auf -o-, germanisch -a-, ausgieng. Das gotische und althochdeutsche lassen den zweiten stamm auf -ai, ahd. $-\hat{e}$ - ausgehn, sie bilden von diesem stamme auf -ai- das part. pass., das gotische ausserdem sein jüngeres verbalsubstantiv auf -ni-, das bei den schwachen verben das alte auf - $t i$ abgelöst hat, got. -ains, wie in der $\bar{a}$-conjugation -ôns. Das gotische und hochdeutsche haben aber das -ai- aus -o-je- der 2. 3. sing., 2. plur. präs. zum verbalstanm erhoben. In den ubrigen germanischen dialecten findet sich von diesem -ai- im präteritum keine spur. Von den auf -o-, germ. -u-, ausgehen-

1) Das dïnische besitzt noch einen wirklichen infinitiv dieser klasse in turde, zu an. pora, priit. porda. - Der nordfries. dialect der insel Sylt verwendet ausser von den präteritopräsentien auch von dem zu unserer klasse gehörenden verb 'haben' formen die den participien des passivs gleichlautend sind in infinitivischem sinne nach bestimmten syntaktischen regeen, doch ist es nicht sicher, ob in diesen formen alte infinitive auf -ti-stecken, oder die participien mit ellipse des infiuitivs 'haben'.

2) Vgl. zum folgenden Bezocuberger, G(xA. 1si9, stiick 29, s. 91s ff. 
den \%weiten stamme ist das prät. anzuset\%en als urgernan.

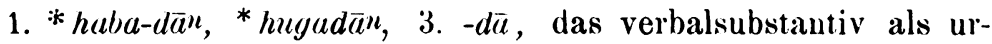
germ. *pula-di-s, *hugadi-s. Diese formen haben wir zu grunde zu legen: von ihnen müssen wir suchen zu den uns vorliegenden habda etc. zu gelangen.

Die nordischen formen fügen sich den vorausgesetzten ohne weiteres, sämmtliche nordischen präterita unserer klasse können nach den uns bekannten lautgesetzen aus jenen formen - mit mittlerem a entstanden sein. Was aber geschieht mit mittlerem $a$ in unbetonter silbe in den ubrigen germ. dialecten? Wie im englisch-friesischen auslautendes $i$ und $u$ und mittlerer vocal $i$ und $u$ gleich behandelt werden, so wäre $z u$ erwarten dass auch mittlerer vocal $a$ wie end- $a$ behandelt werde, also habadä'n wie dagaz ésti. Nach sievers untersuchung, Beitr. V, s. $70 \mathrm{ff}$, in welcher mittlerer vocal $a$ von $e, i, u$ nicht getrennt wird, ist dies aber nicht der fall: mittlerer vocal $a$ bleibt nach kurzer silbe. Das germanisch, das sievers hier voraussetyt, stimmt in bezug auf die mittelvocale im ganzen zum gotischen. Das gesetz, nach dem die $a$ in ${ }^{*} h a b d a,{ }^{*} h u g d a$, wie in got. gahugds geschwunden sind, muss vorgotisch, es muss ein allen germanischen dialecten gemeinsames gewesen sein. Es scheint mir die folgende ausdehnung gehabt zu haben: 'Mittleres a vor einfachem geräuschlaut schwindet in der gruppe $\smile \smile '$, , die dadurch ${ }^{\prime}$ wird, d. h. 'urgerm. $a$ in der zweiten von zwei unbetonten kurzen silben schwindet vor folgender hochbetonter silbe, die mit einfachem verschluss- oder reibelaut aulautet.' Der ausfall des $a$ ist damit in die zeit vor der germanischen accentverschiebung verlegt. ${ }^{\text {') }}$

1) Im gotischen widersprechen der regel nur zwei fälle: 1)/irumadei i., eine jüngere ableitung von einem *frumaps, das den ton vorgermanisch auf dem $r$ getragen haben kann, und 2) gagudalıa und gatilalı, die beideu einzigen kurzsilbigen arlverbien aut - ba, wenn dies -ba urspriinglich -ku'd ist, ablativ der iu slavischen so gewöhnlichen weiterbildung der adjective durch $-k \bar{u}$ (got. hardu-lu wie griech. $\vartheta \eta \lambda v-\varkappa \omega_{s}$ ): die kurzsillbigen können sich hier der überwältigenden mehraahl der langrsilbigen gefü̈rt haben.

$\smile \dot{\smile} \smile$ waren ursprünglich die -ap- wie dalap, samap, magaps,

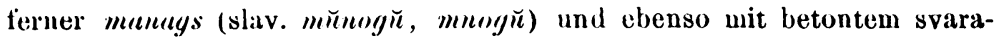
bhaktivocial wol araks $(=$ skr. $a i z a s$, also jedenfialls mit mbetontem 
Urgerm. *habadā'n ward, wenn diese fassung des gezettr's richtig ist, regelrecht $h a b d \bar{a}^{\prime} n$, as. habda etc., das verbalswub-

vocal der urspr. endsilbe). $\doteq \smile こ$ waren die obliquen casus des consonantischen stamms mitad-f., gen. pl. miladê, die passivformen bairaza, bairada, forner ahaks, nakvaps (germ. nikuada-z = lat. nülus uit dem suffix lat. -ido-, dessen $d$ urspr. $d h$ ). Unser ältestes germanisches lehnwort, herübergenommen vor der lautverschiebung, hanapi-z hanf, trug nach griech. $x \alpha \dot{v} \alpha \beta \iota \varsigma$ den accent auf der ersten silbe. Durch position war das mittlere a geschützt in alakjô, billagva, den wörtern aut -rlvawie bivadv, den verben auf -atjan, wie dem von unserm verbalstanm huga- abgeleiteten ahd. hogazzen, deren -tja- $=$ urspr. -d-jo- (micht -déjo-), s. s. 4 io.

Dass ein gesetz iiber den ausfall eines $a$ von der art des oben formulierten bestanden haben muss, lehren die urgermanischen auf ein $-a$ auslautenden proclitischen wörter der gestalt $\smile \smile$. Enclitische und proclitische wörter werden mit dem worte, an welches sie sich anlehnen, als éin wort behandelt. Jene $\smile \smile$ nun haben wo sie proclitisch sind, ihr auslautendes - $a$ gemeingermanisch eingebiisst und sind einsillig geworden. Es kann angenommen werden, wenn das gesetz seine obige enge fassung behalten soll, dass der ausfall nur vor unmittelbar folgender hochtoniger silbe mit anlautendem einfachen geriuschlaut erfolgt sei, dass dann aber das so entstandene cinsilbige proclitische wort sein geltungsgebiet erweitert habe.

Das erste wort der ältesten auf uns gekommenen germanischen inschrift des Hlevagastiz lautet $e k$, während anf derselben in horna und gleichzeitig in staina der ausgang germ. -a aus urspr. -om sich hält. Nach sanskr. ahám lautete das pronomen wo es vollbetont war germ. eká : in 'ek Hlévagastiz' etc. aber war das pronomen, wie das metrum zeigt, proclitisch, und als solches entbehrte es vor hochtoniger silbe des -a. Dasselbe geschah mit den prïpositionen germ. af $a=$ skr. ipa gr. "̈ ( $\left.\alpha^{\prime} \pi \dot{o}^{\prime}\right)$, germ. $a n a=\mathrm{gr}$. $\alpha^{\prime} \nu \dot{\alpha}$, die proklitisch $a t$, all wurden. Ich glaube nicht, dass Sievers recht hat, wenn er a.a. o. s. 12. lehrt, dass urspriunglich auslautendes unbetontes $a, e, i$ bereits in der germanischen grundsprache abgefiallen sei. Denn nur die durch unser geset's liber den ans. fall des $a$ entstandenen einsilbigen wörter verfallen gemeinsam mit den ursprünglich einsilbigen im friesisch-englischen einem bestimmten lautgesetz: sie erleiden dehnung des vocials.

Ursprünglich einsilbige wörter erfahren regelmässig dehnnng des vocals: altfiries. hwâ wer, hî er, thì du u. s. w., ausserdem weisen die friesischen mundarten auf altfries. $e t$ bei, thêt das u. dgl., die entsprechende dehnung ist auch für das englische nachzuweisen. (Genaueres \%u diesem und dem folgenden in meiner nordfries. gramm.).

Dieselbe dehnung widerfïhrt den firiher zweisilbigen ant $a$ der ursprünglichen gestalt $\smile \smile$. $\hat{\imath} c$ ich, $\hat{o} n$ an, $\hat{\text { f }}$ ab sind die formen, die in den altenglischen die quantität bezeichmenden handschriften constant 
stantiv urgerm. * hugadi-s, * puludi-s regrelrecht hugdi-s, puldi-s, got. -higds, as. -thuld.1)

Dé präterita unserer klasse, und also der schwachen verben iiberbaupt, können nach dem dargelegten so gut $t$ - wie dh-präerita sein.

De participien der alten $o-$, germanischen $a$-klasse, scheine vorgerm. die gestalt $-\bar{o}-t \dot{o}-s$ gehabt zu haben, im lateinischen nach Curtius bewahrt in aegrōhs (nit der dehnung des aulautenden vocals des verbalstamms, tiber welche Bezzenberger a. a. o.). Im nordischen haben alle participien der $a$ klasse diesen ausgang bewahrt, an. aðr neutr. -at. Auch hafat, sagat snd die älteren formen: wenn von diesen verben das part. alch nach der analogie des präteritums gebildet wird, haft, sugt, so ist der grund nicht der von Paul angenommene, dass gsrade diese verben ursprünglich ihr prät. und part. vocallos bildeten, sondern der grund ist (obwol auch spartr vorkomont) das in diesen verben eingetretene $e$ des präsens, 1. sing. hefi, segi und ebenso begi, inf. segja, beg.ja, welches

zu finder sind. Die lïnge des vocals lässt sich noch aus englischen nundarten erweisen. Fiir die entsprechenden friesischen wörter beweisen siimiliche ost- und nordfriesische mundarten die länge des vocals. Wo die rocale in englischen mundarten kurz sind, wie in ne. on, of (at, that), da ist dies reguläre kürzung des vocals, bewirkt entweder durch preclisis oder durch die folgende consonantengruppe (den ausliutender. cons. und den anlautenden des folgenden wortes). Fries. engl. $" \hat{\imath} l$ alv. Wol (ne. schrittspr. well, gegenüber dem weel zahlreicher mundarten) = an. vel as. wel, kann ein neutrum auf urspr. -om sein, gedehnt wo unbetont vor folgender hochbetonter silbe.

1) Urspriingliches $m+l$ wird durch mpt germ. $m f t$, durch den ausfill dès a idisanmengerlicktes $m+d$ aus $t$ aber wird vielleicht $n d$ in stimula f'. (aus -o-tii'), wenn dies zu dem verb unserer klasse skaman gehört. (I)je nordischen präterita auf -mola können sehr wol durch die allalogie des präsens wider zu ihrem "' gekommen sein, vielleicht aber ist dieser iiberyang des $m$ in $"$ dem nordischen von haus aus fremd: wird vielleicht urgermanisch zusammengeriicktes $m p$ vor der tonsilbe zwar gotisch! und westgermanisch $\mathrm{ud}$, aber nordisch $\mathrm{mm}$ in siomm f.? Ein nordisches präteritım -mma konnte nicht anders als durch -mda sich ersetzen lassen, wic olla sich von olda ablösen liess.) - Germ. zd wird auch durch zusammenriickung nach ausfall eines $a$ haben entstehn kiinnen, so vielleicht in razda fi. stimme (skr. wurzelverb risati laute von sich geben, tünen). 
da\%u verfibite das part. wic in der ersten schwachen conj. zu bilden, sagt, bagt. Den alten participialausgang unserer klasse hat im nordischen auch das part. zu hyggja bewahrt, hugat, und in adjectivischer bedeutung hugadr (das $u$ in prät. hugja, part. hugat nach der ersten schwachen conj.): ein sicherer beweis, dass wir recht hatten das prät. *hugda als form der schwachen $a$-conjugation zu erklären. Im altengl. ist der alte participialauscang gewahrt in žehozంd: dieses -od und die analogie der 3. schwachen conj. verführten dazu, auch das prät. auf -ode zu bilden, hozod', polode und so bei zahlreichen ursprünglich zu unserer klasse gehörigen verben. Von haus aus kam dies -od, und danach -ode, nur den verben der zweiten, dagegen -ade, -ad den verben der dritten schwachen, urspriinglichen $\bar{a}$-conjugation zu, natürlich gehn diese formen dann aber in einander.1) Die participien as. gisagd, ebenso ae. zesa zd, -lifd sind nach der analogie des präteritums gebildet, wie dieses abhängige verhältnis des particips zum präteritum iiberhaupt iiberall das reguläre ist. Got. hafts, in dem Paul s. 148 das alte, vocallos gebildete participium sieht, fitr urspringliche genossen * sahts, *lifts zeugend, ist gar nicht part. von hahan, sondern von hafjan, denn es ist = lat. captus.

Den eausativen verben der rerm. ersten schwachen conj. mit dem präsensstamme auf $-i-j o-\mathrm{kam}$ im zwciten stamme und im particip ursprïnglich durchaus ein $-i-z u$, prït. germ. $-i-d \bar{a}^{\prime \prime}$, part. $-i-d a-z$. Consonantisch auslautenden zweiten stamm hatten im gemeingermanischen, wie wir sahen, von verben auf -éjo- nur pankjan und lrangjan, deren analogie im westgermanischen die causative auf -akjan und -aljan sich anschlossen. Alle andern an einzelnen punkten des westgermanischen gebiets auftauchenden, anscheinend ursprbinglich zweisilbigen priiterita und participien zu causativen verben sind junge neubildungen.

Das lagila des Cott. (nehen legda des Mon., s. Begremann

1) Ein in substantivischem gebrauche erhaltenes particip anf -odazu einem verb unsrer schwachen a-conjugation ist got. vitôf ahd. wizzit n. zum schwachen verb got. vitan. Das niederlaind. wet f. gesetz ist dagegen urgerm. vitadio', eine bildung wie sie eben fiir skanda vermutet ward. 
s. 121) ist absolut nichts anderes als eine junge analogiebildung nach sagda: seggian sagda, folglich leggian lagda. Das mittelfrünk. satte, latle, ndfiänk. (psalm.) satıu, des Cott. satıa, lalta, Mon. einmaliges quadda (sonst quedda wie im Cott. immer, s. Begemann) sind ebenso, wenn auch aus der zeit vor der verschiebung des $t$ zu $z$ im prïsens des mittelfränk. datierende, analogiebildungen einerseits nach den etwas älteren prïteriten wie wahta, salda, andrerseits nach habda, sagda.

Das von Paul als nächste vorstufe für das ahd. sazıa, lazta angesetyte * satda, * latda (also mit den verschlusslaut $d$, woraus hd. $t$ ) ist eine unform: ein solches $t l$ hätte sich keinen augenblick lalten können, sondern hätte sofort $t t$ werden müssen. Was aus einem zu grunde liegenden urspriinglichen $d$-dh geworden wäre (germ. $d d$ oder $z d$ ?) können wir nicht sagen, da ein solcher fall uns nicht vorliegt. Ahd. sazta, lazia kann absolut nichts anderes sein als einfach eine bildung des präteritums zu sezzan nach der analogie der langsilbigen verben, dasselbe, was von den li-verben jüngere formen wie pidachta (B.R.) sind. Vgl. Paul selbst, Beitr. VI, 152. Ebenso sind ahd. qualta, mhd. ratte, tratte analogiehildungen nach den langsilbigen verben. Ihnen entsprechen auf niederdeutschem gebiete die zweisilbigen formen mit dem vocal $e$, setta. letta, quedda, legda. Endlich sind ebenso auf englischem boden die ae. sette, lette, hwette, lredde, ahredde nur analogiebildungen nach den langsilbigen: als solche sind auch wol einfacher und richtiger dic oben geschenen wehte, pehte, rehte anzusetzen, da auch im prät. und pirt. der ursprtinglich langsilbigen verben auf germ. kk das $c t$ lic umbildung zu $h$ nach der analogie urgermanischer $h t$ mitmacht. Pauls einwendung, dass im präsens der wechsel von cc tiud $c$ noch gewahrt ist, schliesst eine analogiebildung nach den langsilbigen keineswegs aus, denn mit der analogiebildung ward eben auch jener wechsel aufgegeben: zu recest gehört renhle, dagegen reccest zu rehte, settest setst etc. zu sette. Die formen mit dem umlaut ae. hwette, hredde, tredde sind nicht in stande, lirspribiglich obne vocal gebildete * hwatda, * hradda, * tradda zu erweisen, welche Paul ihnen s. 148 entnimmt, allerlings dieselben mit einem * versehend, fiir sein verzeichnis der entschieden für idg. $d$ h sprechenden 'mit bestimmtheit als von alters vocallos vorauszusetzenden präterita'. 
Stellen wir seinem verzeichnis ein anderes entgegen.

Wir haben im germanischen 1) folgende präterita, die so gut ursprüngliche 1- wie $d h$-präterita sein können: skulda, nunda (nach diesen das junge vilda); ${ }^{*} s \bar{e} d a,{ }^{*} k n \bar{e} d a$ etc. (an. síða, ahd. sàta ctc.); die präterita der schwachen a-conj. hugda, libda, habda, sagda, *bulda (s. 474); sänmtliche reguläre -da der schwachen conjugationen und die jüngeren westgermanischen salda, talda, kwalda.

2) Folgende, die nur $d h$-präterita sein können, sonst junge analngiebildungen sein müssen: die niederdeutschen und mittelfränkischen satta, latta, quadda, lagda.

3) Folgende endlich, bei denen die frage lautet ' $t$-präteritum oder analogiepräteritum': kunpa, unpa, -gunpa, vulpa (an. olla); vissa, mōssa; dorsta, porfta, mahta, aihta, nohta, dohta; brûhta, sōhta, rōhta, bohta, vorhta, pûhta $\left.{ }^{1}\right)$; das nur im gotischen erhaltene (kaup)-asta; pâhta, brâhta, und die westgermanischen wahta, pahta, rahta.

Diese letzten präterita auf $p, s s, s t, f t, h t$ stehn von den ältesten zeiten her, soweit unsere iberlieferung reicht, fest: jene satta etc. begegnen nur auf einem engen gebiete und herschen auch auf diesem nicht ausschliesslich, sondern ringen mit andern bildungen um die oberhand. Wer sich auf die seite jener satta stellt, der hat allen grund, mit Paul (s. 142) auf das gotische zu schelten, lass es 'auch hier wie so vielfach am allerunsprunglichsten ist': sehen wir aber genau zu, dann erkennen wir, dass das gotische doch nicht so gan\% systemlos verfahren ist. Nur jene lagda, satta, latta verleugnet es, noch weniger ist ihm Pauls *salda, *latda bekannt, dagegen alle sicher altgermanischen präterita der dritten reihe, lie mit entschiedenheit auf urspr. $t$ hinweisen, hat es festgehalten, soweit die formen uberliefert sind, mit einziger ausnahme von sôhta (die fälle, wo das gotische das perfect dem prät. vorgezogen hat, got. du-gann, vaivald, sind hier nicht mit zu nennen). Dass

1) forhta ist hier nicht mit anzusetzen, weil das $t$ auch dem prïsensstamm zusteht. Das älteste prïsens ist ahd. forahtan, ein starkes verb mit dem präsensstamm auf $-t \dot{o}^{-}$. S. Engl. stud. III, 161. Das prït. forhta enthïlt nicht etwa ein $t+t$ oder $d h$. Got. faurhtjan ae. a-fyrhtan ahd. furihten ist ein schwaches verb, zahlreichen andern der gleichen bildung völlig analog, dem jenes prät. urspriinglich fremd ist. 
das gotische die formen habda, libda nicht gewahrt hat, kann ihm nicht vorgeworfen werden, da die richtung, welche die gotische sprache einschlug, nun einmal dahin gieng, dem prät. dieser conj. die gestalt -aida zu geben: das mit seinem präsens analogielos dastehende hugda aber vermochte es naturlich nicht zu halten, sondern muste $\mathrm{cs}$, nach dem präsens, hugida werden lassen.

Als $t$-präterita betrachtet haben die präterita auf $b, s s$, $f t, h t$ las ihnen gebuhrende particip neben sich, soweit es von der sprache gebildet ist. Als $d h$-präterita betrachtet haben dagegen die satta, latta, quadda, lagda ibr ihnen gebuhrendes particip nicht, und die anhänger des $d h$-präteritums muten uns darum $\% u$, bei diesen das particip (mfrk. gesat, gelat ${ }^{1}$ ) als analogiebildung nach dem präteritum, wie bei den präteriten auf $b, s s, f t$, ht das präteritum als analogiebildung nach dem particip anzusehen: das ihnen von rechts wegen gebuhrende part. gewinnen jene satta, latta erst, wenn wir sie als junge analogiebildungen nach salda mit dem part. gisald ansehen.

Nach allem diesen ist es mir keinen augenblick zweifelhaft, welcher der beiden reihen, der kurzen satta, latta, quadda, lagda, oder der langen von kunpa bis brâhta, der preis des alters zu erteilen, und welche andere fur eine jungere aualogiebildung zu erklären ist. Meine antwort auf diese frage steht zu der von Paul gegebenen in directem gegensatze.

1) heass ist (gegen Paul s. 14S) nicht das part. zu Pauls *hwatda, d. h. zu dem vom allj. an. hvalr as. hwat ahgeleiteten verb hvatjan, sondern wie Kägel 8. 175 richtig ansetzt. zum st:urken verh * hvalan * huit $(a=A)$. Fis stellt sich zu den von Paul s. 14! anm. 2 aufgefiihrten participien alds zu alan etc.

KIEL.

HERMANN MÜILLER. 